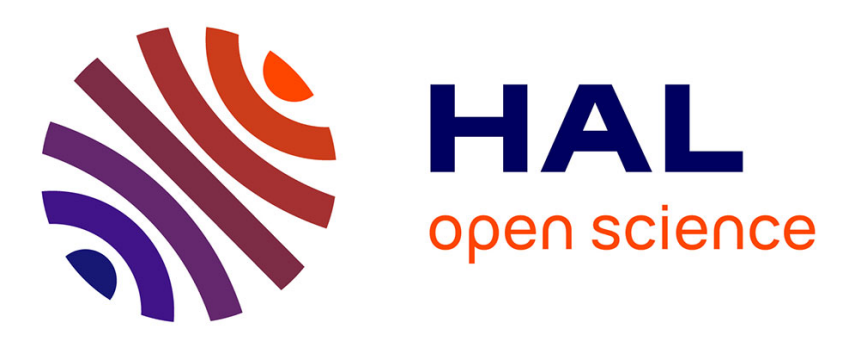

\title{
One-day measurement to assess the auditory risks encountered by noise-exposed workers
}

Thomas Venet, Pierre Campo, Cécile Rumeau, Aurélie Thomas, Cécile Parietti-Winkler

\section{- To cite this version:}

Thomas Venet, Pierre Campo, Cécile Rumeau, Aurélie Thomas, Cécile Parietti-Winkler. One-day measurement to assess the auditory risks encountered by noise-exposed workers. International Journal of Audiology, 2014, 53 (10), pp.737-744. 10.3109/14992027.2014.913210 . hal-01070835

\section{HAL Id: hal-01070835 \\ https://hal.science/hal-01070835}

Submitted on 7 Oct 2014

HAL is a multi-disciplinary open access archive for the deposit and dissemination of scientific research documents, whether they are published or not. The documents may come from teaching and research institutions in France or abroad, or from public or private research centers.
L'archive ouverte pluridisciplinaire HAL, est destinée au dépôt et à la diffusion de documents scientifiques de niveau recherche, publiés ou non, émanant des établissements d'enseignement et de recherche français ou étrangers, des laboratoires publics ou privés. 
1 One-day measurement to assess the auditory risks encountered

3

\title{
by noise-exposed workers.
}

\author{
Running title: DPOAE suppression and auditory fatigue
}

\author{
Thomas Venet $^{\mathrm{a}}$, Pierre Campo ${ }^{\mathrm{a} *}$, Cécile Rumeau ${ }^{\mathrm{a}, \mathrm{b}}$, Aurélie Thomas ${ }^{\mathrm{a}}$, Cécile Parietti- \\ Winkler ${ }^{b}$.
}

\author{
${ }^{a}$ Institut National de Recherche et de Sécurité. Rue du Morvan. CS 60027. F-54519
} Vandœuvre Cedex. France; Tel: 333835021 55; Fax: 333835020 96;

Pierre.campo@inrs.fr

\author{
${ }^{\mathrm{b}}$ Service O.R.L. et Chirurgie Cervico-faciale CHU-Hôpital Central, Nancy, France \\ ${ }^{\mathrm{b}}$ INSERM U954, Faculté de Médecine, Nancy-Université, France
}

\section{Acronyms}

CAS DPOAE: Contralateral acoustic suppression of DPOAEs dB HL: Decibel hearing level dB SPL: Decibel sound pressure level DPOAEs: Distortion product otoacoustic emissions $\triangle$ DPOAEs: [DPOAEs at the end of the shift - DPOAEs at the beginning of the shift] DP-gram: DPOAE pattern across frequency $\triangle \mathrm{DP}$-gram: [DP-gram at the end of the shift - DP-gram at the beginning of the shift] ER: efferent reflex $\triangle E R$ : [ER threshold at the end of the shift - ER threshold at the beginning of the shift] INRS: Institut National de Recherche et de Sécurité; French Research and Safety Institute for the Prevention of Occupational Accidents and Diseases LEX,8h: equivalent continuous noise level calculated over $8 \mathrm{hr}$ MER: Middle-ear reflex NIHL: noise-induced hearing loss PTA: Pure-tone air-conduction audiometry $\triangle$ PTA: [PTA at the end of the shift - PTA at the beginning of the shift]

${ }^{*}$ Corresponding author 


\section{Abstract}

Noise is one of the most pervasive hazards in the workplace. Despite regulations and preventive measures, noise-induced hearing loss is common. The current reference test is pure-tone air-conduction audiometry (PTA), but this test cannot be used to detect early hearing loss. OBJECTIVE: In this study, we assess one-day auditory fatigue using both PTA and efferent reflexes (ER) measured using DPOAEs associated with contralateral acoustic stimulation (CAS DPOAEs). DESIGN: The noise exposure history, PTA, and ER detection were performed in seven different companies where the $\mathrm{L}_{\mathrm{EX}, 8 \mathrm{~h}}$ was $85 \mathrm{~dB}(\mathrm{~A})$. Hearing was tested before and at the end of the working day. STUDY SAMPLE: 46 volunteers were selected to carry out this study. RESULTS: After a single working day, a greater impact of noise was measured using ER thresholds than PTA or DPOAEs. ER measurements are objective, easy to perform, and do not require a sound-attenuated booth. CONCLUSIONS: Screening workers by periodically measuring ER thresholds using CAS DPOAEs helps detect early changes in hearing status, before the onset of noise-induced hearing loss. These tests can be readily applied as part of a hearing conservation program.

(1) 
62

\section{Introduction}

Despite extensive regulations and preventive approaches, noise-induced hearing loss (NIHL) remains a major occupational health hazard in industrial environments (Nelson et al., 2005). In 1996, the NIOSH established the National Occupational Research Agenda (NORA). This identified NIHL as one of the 21 priority areas requiring improvement in occupational practices. Hearing loss is not restricted to the civilian workforce; it is also a significant public and military health problem. From a general point of view, NIHL can be defined as permanent auditory threshold shifts based on measurements performed using pure-tone airconduction audiometry (PTA). Temporary auditory threshold shifts (TTS) also exist, these can be considered as auditory fatigue. In the current study, we mainly studied the auditory fatigue to evaluate the risk encountered by workers exposed to moderate-intensity noise.

In most hearing conservation programs, the auditory performances of noise-exposed workers are tested using PTA. PTA relies on a patient's ability to detect thresholds of hearing sensations based on frequency-specific acoustic stimuli, making it a subjective test. In this kind of test, the central auditory system analyzes each piece of information provided by the peripheral auditory receptor, and may compensate for subtle ear dysfunctions or metabolic fatigue to ensure the highest level of performance. Such phenomena are part of the general concept of plasticity of the auditory function (Syka, 2002; Kaltenbach \& Zhang, 2007; Finlayson \& Kaltenbach, 2009; Mulders \& Robertson, 2013). Thus, retro-cochlear compensation mechanisms may lead to underestimation of temporary auditory threshold shifts (fatigue), and this can postpone the identification of changes in hearing capacity.

For many years, people responsible for noise prevention have been hoping for a rapid, objective test. Today, clinicians, and some occupational physicians, measure distortion product otoacoustic emissions (DPOAEs) to detect inner-ear dysfunctions due to loud noises 
or presbycusis (Lonsbury-Martin et al., 1991; Oeken et al., 2000; Neely et al., 2009). This technique has the advantages of assessing the performances of peripheral auditory receptors in the absence of central influences. When two-tone stimulations at $\mathrm{f} 1$ and $\mathrm{f} 2$ (primaries) are emitted simultaneously into the outer ear canal, several DPOAEs can be measured. The most robust of these is the cubic difference, measured at $2 \mathrm{f} 1-\mathrm{f} 2$. This requires a frequency-selective compressive nonlinearity in the basilar membrane mechanics for the region where the primaries overlap (Ruggero et al., 1997; Lopez-Poveda \& Johannesen, 2009). This nonlinearity is mainly due to motility of the outer hair cells (Davis, 1983; Dallos, 1992), which are particularly sensitive to noise-induced damage (Hamernik et al., 1989; LonsburyMartin et al., 1993).

The current study aims to find an appropriate hearing test to monitor auditory fatigue. This test should better estimate the hearing risks encountered by workers exposed to moderate-intensity noise during their working day $\left[\mathrm{L}_{\mathrm{EX}, 8 \mathrm{~h}}=85 \mathrm{~dB}(\mathrm{~A})\right]$. The objective was not to replace PTA, but rather to complement it with a battery of tests including DPOAEs, and DPOAEs combined with a contralateral acoustic stimulation (CAS DPOAE). In this experimental context, PTA thresholds were measured to evaluate overall auditory performance, DPOAEs were used to assess the function of outer hair cells, and CAS DPOAEs evaluated the efficiency of the efferent reflex (ER). This reflex is considered to be the sum of the effects induced by the stapedial and olivocochlear reflexes. PTA, DPOAEs and CAS DPOAES were measured prior to and after a workday. The battery of tests revealed effects on the outer hair cells, the middle-ear, and the auditory nervous centers (Müller and Jansen, 2008; Wagner et al., 2007; Venet et al., 2011; Marshall et al. 2001).

.




\section{Study Participants}

113 Subjects were recruited from among 79 employees working for 7 companies across different

114 economic sectors (Table 1). All participants were volunteers and gave written consent prior to 115 testing for the inclusion phase. This cross-sectional study was promoted by the ENT

116 department of the university teaching hospital, Nancy, and INRS; it was approved by the national ethics committee (CPP 10.0702, Affsaps UEC/AnnR/DA/2010-212). DPOAE measurements, and (3) ER detection. Subjects for whom no ER could be measured, or those with at least $35 \mathrm{~dB} \mathrm{HL}$ of hearing loss at any tested audiometer frequency, were excluded. Anamnesis was also performed by occupational physicians to exclude workers being treated with ototoxic drugs or those exposed to ototoxic chemicals (Campo et al., 2013). Three workers were excluded because of poor PTA performances, 11 due to weak acoustic DPOAEs, 9 because of a high ER threshold ( $>92 \mathrm{~dB}) \mathrm{HL}), 6$ because of their medical histories, and 4 because of technical problems during noise exposure measurements.

The selected volunteers $(n=46)$ were divided into two groups: a control group of 20 subjects, exposed to $67.3 \pm 4.7 \mathrm{~dB}(\mathrm{~A})$; and a case group (noise-exposed group), consisting of 26 factory workers exposed to $85 \pm 2.9 \mathrm{~dB}(\mathrm{~A})\left(\mathrm{L}_{\mathrm{EX}, 8 \mathrm{~h}}\right.$, details in Table 1). This is the upper limit

129 for noise exposure recommended by the European noise legislation (Directive2003/10/EC)

130 and by the United States legislation (OSHA, 1910/95) before ear protection is required.

131 The mean ages were $36 \pm 8.3$ years old [23,50], and $38.5 \pm 11$ years old $[18,54]$ for the case 132 and control groups, respectively. 


\section{$3 \quad$ Materials and Methods}

137 Workers were seen individually in a special lorry where INRS personnel were authorized to 138 carry out medical research (authorization: SGAR No. 2008-389 obtained on 30th October 139 2008). An occupational physician asked all participants to complete a questionnaire relating 140 to their acoustic and therapeutic histories.

141 3.1. Otoscopy. Otoscopic examination was performed to verify the absence of 142 infection, that the eardrum had a normal appearance (without scarring or perforation), and to 143 ensure that the external auditory canal was not occluded with dry or impacted cerumen.

144 3.2. Conventional audiometry. Hearing acuity was tested by PTA. The audiometer 145 (Interacoustics AS608) was used with a THD39 headphone equipped with Peltor H7A muffs.

146 It was calibrated according to the procedure described in EN 60645-1/AINSI S3.6, type 4.

147 Three audiograms were performed: one for subject selection, one prior to, and one after the 148 working day. Sound stimuli were presented in the following order: 1000, 2000, 3000, 4000, 1496000,8000 , and $500 \mathrm{~Hz}$. The duration of the working day was similar in both case and control 150 groups, with an average of about $7.5 \mathrm{~h}$ (Table 1). Examinations were performed near the 151 workplace so that hearing tests could be conducted within a few minutes before starting and 152 after (<5 min) completing work. PTA was the first test performed in all the series of hearing 153 tests. Background noise was at or below the level recommended in standard ISO 8253$154 \quad 1: 2010$.

3.3. Tympanometry. Normal (type A) acoustic compliance was checked in each ear 156 by immittance using a $226 \mathrm{~Hz}$ probe tone with a static pressure change in the external ear canal of $200 \mathrm{daPa} / \mathrm{sec}$ varying from 200 to $-200 \mathrm{daPa}$ (GSI Tympstar, 2000-97XX). 
3.4. Input/Output DPOAE procedure. The DPOAE probe (Etymotic Research

159 ER10C) contained 2 transducers, with a bandwidth ranging from $200 \mathrm{~Hz}$ to $12 \mathrm{kHz}$ at \pm 10

$160 \mathrm{~dB}$. The transducers generated two pure tones: $\mathrm{f} 1$ and $\mathrm{f} 2$, chosen to generate cubic DPOAEs

161 with an f2/f1 ratio of 1.2. The intensities of the primaries were L2 = L1 - $6 \mathrm{~dB}$ HL (Gaskill \&

162 Brown, 1990; Whitehead et al., 1995; Neely et al., 2009). Two synthesizers (Pulse, B\&K

163 3610) were connected to the probe to deliver f1 and f2 into the external auditory canal; the

164 highest intensity was limited to $70 \mathrm{~dB}$ HL to avoid activating the ER. The levels of $\mathrm{f} 1$ and $\mathrm{f} 2$ were emitted in $\mathrm{dB}$ HL, whereas the DPOAE levels were measured and expressed in $\mathrm{dB}$ SPL. An Ear Simulator (RA0045 GRAS IEC 711) was used to calibrate the system according to standards ISO 389-2 and IEC 60318-4.

These two procedures ensured that $\mathrm{f} 1$ and $\mathrm{f} 2$ were always emitted at the target intensities, regardless of the probe used. Moreover, calibration in $\mathrm{dB}$ HL makes it easier to correlate primaries with the intensities used for contralateral noises.

Three couples (f1/f2) of frequencies were tested: (3000/3600), (4000/4800), and

172

(5440/6528) Hz. DPOAEs were elicited in response to stationary stimuli and recorded with a microphone embedded in the probe. The three transducers were enclosed in the probe, the tip of which was inserted into the subject's external auditory canal. A fast Fourier transform (frequency span $25.6 \mathrm{kHz}, 3200$ lines, time-weighting Hanning window, overlap 66.7\%) was applied to the acoustic signal. The instantaneous DPOAE was determined from a linear average of spectra $(\mathrm{N}=4)$, the mean was calculated over $250 \mathrm{~ms}$ with a frequency resolution of $8 \mathrm{~Hz}$. The overall DPOAE level was calculated from 20 instantaneous DPOAEs. For each frequency couple, DPOAE amplitudes were acquired as a function of $\mathrm{f} 1$ and $\mathrm{f} 2$ intensities, which increased from 49 to $70 \mathrm{~dB}$ HL in 3-dB steps. The baseline noise level was calculated by averaging the levels at three neighboring frequencies: the instantaneous DPOAE and the two frequencies located either side of it. For measurements to be acceptable, the signal-to- 
183 noise ratio (SNR) had to be greater than $3 \mathrm{~dB}$ relative to the average calculated on either side

184 of the DPOAE. This test was the second carried out in the series of hearing tests.

\subsection{DPOAE pattern across frequency: DP-gram.}

186 Following the ear examinations and audiograms, participants were screened for DPOAEs

187 across the f2 frequency, ranging from 1008 to $8064 \mathrm{~Hz}$, with a constant stimulation level:

$188 \mathrm{~L} 1=61 \mathrm{~dB}$ HL and $\mathrm{L} 2=55 \mathrm{~dB}$ HL. Note that the measurements were expressed in $\mathrm{dB}$ HL to

189 facilitate comparison with PTA. The DP-gram was performed just after the input/output 190 DPOAE measurements.

\subsection{Determining the efferent reflex threshold.}

A special device specifically designed for CAS DPOAE measurements was used in different workplaces for these measurements. This device is described in detail in Venet et al.

194 (2012) and has been patented (utility certificate $\mathrm{N}^{\circ} 11$ 51529, publication number 2971931). Briefly, DPOAEs were measured in the ipsilateral ear with an Etymotic Research ER10C probe with primaries at (4000/4800) Hz. The previous DPOAE I/O approach assessed the linear part of the intensity-magnitude relationship for each subject. The intensities of the primaries were always chosen in the linear part of the intensity-magnitude curve (Figure 3) so as to obtain $10 \mathrm{~dB}$ SPL DPOAEs amplitudes. Most of the time, the DPOAE amplitudes were saturated at intensities greater than $65 \mathrm{~dB} \mathrm{HL}$ (Figure 3). It was therefore important to determine the level of saturation to avoid overestimating the efferent reflex threshold.

The contralateral acoustic stimulation was delivered through an earphone (Etymotic Research ER4 B) placed in the outer ear canal. The contralateral noise was a narrow $(800 \mathrm{~Hz})$ band noise centered at 1000, 2000 or $4000 \mathrm{~Hz}$. Each burst was synthesized by a B \& K Pulse 3610 , lasted up to 2 seconds, and was emitted at intensities ranging from 65 to $95 \mathrm{~dB}$ HL. 
measurements). Transition values were rejected. The threshold for significance was set at $\mathrm{p}<0.05$. The upper intensity limit was fixed at $95 \mathrm{~dB}$ HL to avoid effects on workers' hearing. CAS DPOAEs were the last measurements performed in the series.

3.7. Checking noise exposure. Workers were tested 15 minutes prior to starting their job. Then, they were equipped with a noise dosimeter (ACOEM WED) which was also a sound level meter. The dosimeter was worn by workers throughout their working day, and was used to determine the amount of noise that the individual was exposed to during the sampling period. The microphone was placed in the worker's hearing zone.

\subsection{Statistical analysis.}

\section{Statistical tests for pre-work data}

Two-way ANOVA (type III) was run to compare data collected from both controls and exposed-subjects before noise exposure. These statistical results are expressed as follows: $\mathrm{F}(\mathrm{dfb}, \mathrm{dfr})=\mathrm{F}-$ ratio; $\mathrm{p}=\mathrm{p}$ value $)$, in which $\mathrm{dfb}$ is the number of degrees of freedom between groups and dfr the residual degrees of freedom. Between-group degrees always corresponded to the number of groups (case/control) -1 .

The F-ratio is the mean square value between groups divided by the mean square value within a group. Post hoc analysis of statistical significance was performed using the Bonferroni method.

\section{Statistical tests to analyze the noise effects}

The relationships between a one-day noise exposure $\left(\mathrm{L}_{\mathrm{EX}, 8 \mathrm{~h}}\right)$ and the variations in (1) DPOAE

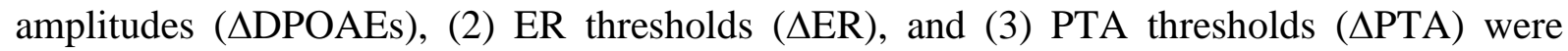
evaluated by applying a standard t-test of differences between controls and noise-exposed subjects. The variables were: $[\triangle \mathrm{DPOAEs}=\mathrm{DPOAE}$ amplitude measured at the end of the shift - DPOAE amplitude measured at the beginning of the shift $], \triangle \mathrm{ER}=[\mathrm{ER}$ threshold at the 
232 end of the shift - ER threshold at the beginning of the shift], or $\triangle \mathrm{PTA}=[\mathrm{PTA}$ at the end of the 233 shift - PTA at the beginning of the shift].

234 


\section{Results}

4.1. Pre-work hearing tests.

4.1.1. Pre-work pure-tone air-conduction audiometry

239 The audiometric curves obtained for both groups before starting work are shown in Figure 1.

240 The workers in the case group were not exposed to any occupational noise over the preceding

24116 hours. Two-way ANOVA indicated there was a significant difference between the case

242 and control groups $([\mathrm{F}(1,195)=4.18 ; \mathrm{p}=0.04]$, but Bonferroni post hoc tests were not

243 significant (contrast difference $=2.04$ with \pm limits $=2.25$ ). The notch observed at $6000 \mathrm{~Hz}$ in

244 both groups was probably due to the noise exposure history of the volunteers.

\section{Insert Figure 1 about here}

4.1.2. Pre-work DP-gram

The DPOAE levels obtained at $61 \mathrm{~dB}$ HL across frequencies before starting work (Figure 2)

showed a similar significant difference between the case and control groups $[\mathrm{F}(1,202)=6.45$; $\mathrm{p}=0.01]$. In this case, Bonferroni post hoc tests were also significant (contrast difference $=-$ 1.75 with \pm limits $=1.55$ ).

\section{Insert Figure 2 about here}

\subsubsection{Pre-work DPOAE input/output}

253 Only data obtained at $(3000 / 3600 \mathrm{~Hz})$ with the DPOAE input/output procedure was 254 significantly different between case and control groups $[\mathrm{F}(1,366)=13.04 ; \mathrm{p}<0.001]$; 255 Bonferroni post hoc tests also showed this difference to be significant (contrast difference = 2562.62 with \pm limits $=1.64)($ Figure $3 a)$.

257 In contrast, at (4000/4800) and (5440/6528) the two groups were not significantly different 258 (Figure 3b,c). The intensity-magnitude DPOAE relationships were approximately linear up to $25961 \mathrm{~dB}$ HL, with a maximum amplitude of $11 \pm 2.5 \mathrm{~dB}$ SPL for (4000/4800). Overall, DPOAE 260 amplitudes started saturating from $61 \mathrm{~dB} \mathrm{HL}$, particularly at $(5440 / 6528) \mathrm{Hz}$. 
4.1.4. ER thresholds

263 The average values capable of triggering the ER in both groups prior to exposure are shown in

264 Figure 4. Although the thresholds were only slightly lower in the control group compared to 265 the case group, the differences were almost significant $[F(1,127)=3.82 ; p=0.053]$. Bonferroni 266 post hoc tests was not significant either (contrast difference $=2.93$ with \pm limits $=3.39$ ).

\section{Insert Figure 4 about here}

The PTA shifts ( $\triangle \mathrm{PTA})$, i.e., the difference in PTA thresholds obtained before and after work are shown in Figure 5 for workers exposed to a $\mathrm{L}_{\mathrm{EX}, 8 \mathrm{~h}}$ of $85 \mathrm{~dB}(\mathrm{~A})$. The differences were maximal at 3 and $4 \mathrm{kHz}$ (4.5 and $3.5 \mathrm{~dB}$, respectively). This was not surprising given the broad noise spectrum involved.

The probability that $\triangle \mathrm{PTA}$ is significantly different as a function of the group (case vs. control) was determined by applying a t-test. The test revealed a significant $(\mathrm{p}<0.01)$ noise effect at both $3 \mathrm{kHz}(\Delta \mathrm{PTA}=5.97 \mathrm{~dB} \pm 1.87 \mathrm{p}=0.004)$ and $4 \mathrm{kHz}(\Delta \mathrm{PTA}=4.95 \mathrm{~dB} \pm 1.51 \mathrm{p}$ $280=0.003)$.

\section{Insert Figure 5 about here}

The DP-gram variations ( $\triangle \mathrm{DP}$-gram) obtained after a single working day are shown in Figure

6. The differences in values were less than $1 \mathrm{~dB}$.

The probability that a $\Delta \mathrm{DP}$-gram is significantly different depending on frequency was as 
$0.42 \mathrm{p}=0.02)$ and $6.5 \mathrm{kHz}(\Delta \mathrm{DP}$-gram $=-0.71 \mathrm{~dB} \pm 0.0 .36 \mathrm{p}=0.05)$. The DP-gram

288 variations at 4 and $4.8 \mathrm{kHz}$ were also close to the significance threshold. The trend was clear 289 but the intra-group variations were too large to reach the 95\% significance level. Although the 290 variation amplitude was lower than that obtained with PTA, the noise-sensitive frequency 291 range was broader, ranging from 1008 to $6528 \mathrm{~Hz}$.

Insert Figure 6 about here

293

294

295 296

\subsubsection{DPOAEs input/output.}

The intensity-magnitude DPOAE relationships are shown for (3000/3600), (4000/4800), and for (5440/6528) in Figure 7a,b,c. The variations in amplitudes of the DPOAEs measured were systematically lower in noise-exposed workers than in controls, except at $67 \mathrm{~dB}$ HL and only for the $6528 \mathrm{~Hz}$ frequency (Figure 7c). However, at this intensity, the intensity-magnitude DPOAE relationship was no longer linear (Figure 3c). Because of this, only the data obtained with intensities from 52 to $61 \mathrm{~dB}$ HL were statistically analyzed. The t-test revealed a significant $(\mathrm{p}=0.04)$ noise effect at $61 \mathrm{~dB}$ HL for primaries $(3000 / 3600 \mathrm{~Hz})$; it did not find any significant differences between the case and control groups for primaries $(4000 / 4800 \mathrm{~Hz})$. In contrast, an overall noise effect was detected between 55 and $61 \mathrm{~dB} \mathrm{HL}$ at $(5440 / 6528 \mathrm{~Hz})$. For instance, the amplitude of variations in DPOAE were $(-1.28 \mathrm{~dB} \pm 0.56 \mathrm{p}=0.03)$ at $55 \mathrm{~dB}$ HL, $(-1.24 \mathrm{~dB} \pm 0.47 \mathrm{p}=0.01)$ at $58 \mathrm{~dB} \mathrm{HL},(-1.25 \mathrm{~dB} \pm 0.42 \mathrm{p}=0.004)$ at $61 \mathrm{~dB}$ HL. The latter couple of primaries was therefore the most sensitive. Based on these results, DPOAEs can be considered to be a frequency-specific analyzer of cochlear dysfunction, with the most sensitive frequency located at around $6 \mathrm{kHz}$.

\section{Insert Figure 7 about here}


4.3.4. ER threshold variations.

314

Figure 8 shows the ER variations $(\Delta \mathrm{ER})$ obtained for both groups. Larger variations in

315 ER thresholds were measured in noise-exposed workers than in controls at the three

316 frequencies tested. According to the t-test, the noise effect was highly significant for all three

317 frequencies: at $2000 \mathrm{~Hz}(4.83 \mathrm{~dB} \pm 1.46 \mathrm{p}=0.001), 4000 \mathrm{~Hz}(5.30 \mathrm{~dB} \pm 1.69 \mathrm{p}=0.002)$ and $3181000 \mathrm{~Hz}(7.26 \mathrm{~dB} \pm 1.64 \mathrm{p}<0.001)$. 


\section{Discussion}

5.1 Hearing performance in study and control groups before work

When the audiometric data from the case and control groups before noise exposure are compared, the curves of PTA thresholds across audiometer frequencies are seen to have different values, although patterns are similar for both groups. A decline in PTA values, varying from 1 to $5 \mathrm{~dB}$, was observed for the subjects in the case group. In addition, the two curves in Figure 1 display a V-shaped notch at $6 \mathrm{kHz}$. Frequencies in the vicinity of $4-6 \mathrm{kHz}$ are the most sensitive to noise in humans. Because this study was not carried out with selected young normal-hearing individuals, but with a cohort of workers from various factories, most of the subjects, including controls, had probably been exposed to broadband noise, at some point in their career (McBride and Williams, 2013). Although the differences between groups were significant, the amplitude variations between groups were quite small $(<5 \mathrm{~dB}$ at 3000 Hz). Overall, the PTA values at 1000, 2000 and $4000 \mathrm{~Hz}$ (ER frequencies test) before commencing work were comparable between groups.

As far as the DPOAEs are concerned, DPOAE input/output curves were significantly different at $(3000 / 3600 \mathrm{~Hz})$. In the same manner, DP-gram amplitudes measured at $61 \mathrm{~dB} \mathrm{HL}$ were lower for the case group than for the control group, specifically at 2000, 3000 and $4000 \mathrm{~Hz}$ (Figure 2). The differences measured between groups for PTA and ER values are at the limit of significance ( $\mathrm{p}=0.042$ and $\mathrm{p}=0.053$, respectively), whereas they are clearly different for DPOAE measurements (significance threshold at 99\% and 99.9\%). These differences in significance between DPOAEs and PTA/ER thresholds could be explained by a central auditory control of peripheral input (Syka, 2002; Kaltenbach \& Zhang, 2007; Finlayson \& Kaltenbach, 2009; Mulders \& Robertson, 2013). PTA and ER thresholds are dependent on central control, whereas DPOAEs reflect only the function of the peripheral receptors (Avan \& Bonfils, 1993). 
5.2 Hearing performance in control and study groups after the working day

350 Although subjects were only exposed to moderate industrial noise: $\mathrm{L}_{\mathrm{EX}, 8 \mathrm{~h}}=85 \mathrm{~dB}(\mathrm{~A})$, DPOAEs and PTA thresholds were sensitive enough to detect slight changes in hearing performance. The differences [ $\Delta$ PTAnoise $-\Delta$ PTAcontrol] at $3 \mathrm{kHz}(5.97 \mathrm{~dB})$ and $4 \mathrm{kHz}$ (4.96 dB) were large enough to reach a significance level of 99\% (Figure 5). At $6 \mathrm{kHz}$, the dip on the audiometric curve could have masked the effects of auditory fatigue. Thus, it would be easier to assess auditory fatigue in subjects with preserved hearing; and conversely, when the frequencies are extensively damaged, auditory fatigue would be difficult to assess at these particular frequencies.

After a workday, variations obtained with both DP-gram and PTA procedures were significant $(\mathrm{p}<0.05$ and $\mathrm{p}<0.01$, respectively). However, the DPOAE procedure assessed a wider noise-sensitive frequency range [1008, 3600 and $6528 \mathrm{~Hz}]$ than the PTA [3000 and $4000 \mathrm{~Hz}]$.

In addition, the DPOAE input/output measurements performed at $(5440 / 6528 \mathrm{~Hz})$ showed a decrease in amplitude at all intensity levels. In fact, this pair of primaries seems to

364 be the most sensitive to the noise exposure during a single working day. This effect is not 365 revealed by PTA thresholds. The different experimental approaches (PTA, DP-grams and DPOAEs) could assess shifts in hearing performance, but none was strikingly more sensitive. PTA and DPOAEs could be complementary in a test battery.

The present results indicate that the relevance of the hearing test depend on the hearing 369 status of the cohort studied prior to measurements. For these reasons, in contrast with Job et 370 al. (2009) and Seixas et al. (2013), who studied young normal-hearing subjects, due to the 371 variations in hearing performance with age and exposure, we are wary of recommending DPOAEs to detect TTS for workers. The differences between the tests observed here and the results described elsewhere could have various explanations. 
First, PTA is the result of suggestive perceptions. Each component of the auditory system (from receptor to cortex) is partly responsible for the threshold shifts. This mainly concerns the inner hair cells, the function of synapses along the auditory pathways, and the retro-cochlear mechanisms involved in the overall hearing process. In contrast, DPOAEs mainly reflect the function of the outer hair cells, and thereby of the cochlear amplifier (Dallos, 1992). Thus, by measuring DPOAEs, only the effects on the cochlea are assessed.

Second, in our study PTAs were always performed prior to DPOAEs in a quiet room. The period during which the PTA measurements were performed (approximately $5 \mathrm{~min}$ ) could be considered as a recovery time. Since recovery after exposure to noise displays a logarithmic function (Laroche et al., 1989), even 5 min can make a significant difference. DPOAE measurements were therefore measured in subjects who had partly recovered from exposure to noise. This, in addition to the differing age-profiles, could explain why our data do not concur with those reported by [Job et al. (2009) and Seixas et al. (2013)].

Finally, DPOAE recordings require a probe to be inserted into the external auditory canal. When conducting experiments with follow-up measurements, the probe position may vary slightly, despite the good reproducibility of our measurements: at $3600 \mathrm{~Hz}, 0.71 \mathrm{~dB}$ and at $4800 \mathrm{~Hz}, 0.77 \mathrm{~dB}$ (Venet et al., 2012). The approach could therefore be improved by developing a method allowing the ear probe's position to be maintained constant during subsequent measurements (Müller and Janssen, 2008).

\subsection{Hearing performance evaluated by ER thresholds}

The most striking findings in this study were the shifts in the ER thresholds after a noise exposure at a $\mathrm{L}_{\mathrm{EX}, 8 \mathrm{~h}}$ of $85 \mathrm{~dB}(\mathrm{~A})$ (Figure 8). This is not the result of a decreased DPOAE due to the inter-aural acoustic stimulations (CAS) since several subjects with unilateral deafness did not show contralateral suppressions of the DPOAEs. This observation argues against transcranial transmission of the contralateral stimulus. 
Exposure to noise during a working day can provoke sensory (organ of Corti) and

401 neural fatigue (afferent and efferent pathways) associated with fatigue of the middle-ear muscles. While the PTA highlights dysfunctions of the inner hair cells and afferent pathways, and DPOAEs assess outer hair cell motility, the ER measured with CAS DPOAEs gathers all these effects into a single series of measurements. Because of this characteristic, the measurement of the ER with CAS DPOAEs appears to efficiently evaluate the auditory fatigue after a workday. This might be due, at least partly, to the physiological and anatomic support of the ER. Indeed, the ER can be triggered by stimulation of the medial olivocochlear bundle, which can modify either the micro-mechanical parameters of the outer hair cells, or contraction of the MER, or a combination of the two. Due to the shape of the depressive effects (data not shown), and the frequency $(1 \mathrm{kHz})$ at which these effects were best observed, the MER might be the major contribution to the suppressive effect, although a medial olivocochlear effect could not be ruled out. Whatever the nature of the ER, the variations observed were large and significant, varying from $4.82 \mathrm{~dB}$ at $2000 \mathrm{~Hz}(\mathrm{p}=0.001)$ to $7.25 \mathrm{~dB}$ at $1000 \mathrm{~Hz}(\mathrm{p}<0.001)$. Consequently, CAS DPOAEs can readily be used to assess auditory fatigue after a single working day.

\section{Conclusions}

Since it is impossible for individuals to detect their own early noise-induced hearing loss, tests must be performed. Among the tests available: PTA tests are time-consuming to perform, subjective, and require specific acoustic conditions. Although DPOAEs can be measured in less demanding acoustic conditions, they are no more sensitive than PTA, especially when used for the follow-up of a population of workers who have already had a lengthy career. CAS DPOAEs can be used to test the inner/outer hair cells and the ER, providing objective measurements that do not require subject participation. This test is quick and noninvasive and can be carried out in a quiet room (nurse's station or meeting room). For 
426 all these reasons, occupational physicians could easily use CAS DPOAEs to monitor the 427 hearing of an at-risk population in the workplace. Longitudinal follow-up will be required to 428 clarify the advantages of CAS DPOAEs in terms of sensitivity to early manifestations of noise 429 insults, or their utility in predicting future hearing loss.

\section{Acknowledgments}

432 The authors would like to thank Aurélie Rémy and Pascal Wild, statisticians at INRS, who 433 designed and performed all the statistical analyses.

434

435

\section{Conflict of Interest}

436 This research was totally funded by INRS. The authors report no conflict of interest. 


\section{References:}

439 Avan, P., Bonfils, P. 1993. Frequency specificity of human distortion product otoacoustic 440 emissions. Audiol, 32,12-20.

442 Campo, P., Morata, T., Hong, O. 2013. Chemical exposure and hearing loss. Disease-a-Month $44359,4,119-138$.

Dallos, P.1992. The active cochlea. J Neurosci, 12(12), 4575-85.

Davis, H. 1983. An active process in cochlear mechanics. Hear Res, 9, 79-90.

Directive 2003/10/EC. On the minimum health and safety requirements regarding the exposure of workers to the risks arising from physical agents (noise) Official journal of the European Communities L042, 38-44.

Finlayson, P., Kaltenbach, J. 2009. Alterations in the spontaneous discharge patterns of single units in the dorsal cochlear nucleus following intense sound exposure. Hear Res, 256, 104117.

Gaskill, S., Brown, A. 1990. The behavior of the acoustic distortion product $2 \mathrm{f} 1 \mathrm{-}-\mathrm{f} 2$ from the 458 human ear and its relation to auditory sensitivity. J Acoust Soc Am, 88, 821-839.

460 Hamernik, R., Patterson, J., Turrentine, G., Ahroon, W. 1989. The quantitative relation 461 between sensory cell loss and hearing thresholds. Hear Res, 38, 199-212. 
463 Job, A., Raynal, M., Kossowski, M., Studler, M., Ghernaouti, C.et al., 2009. Otoacoustic 464 detection of risk of early hearing loss in ears with normal audiograms: A 3-year follow-up 465 study. Hear Res, 251,10-16.

466

467 Kaltenbach, J., Zhang, J. 2007. Intense-sound plasticity in the dorsal cochlear nucleus of rats:

468 Evidence for cholinergic receptor upregulation. Hear Res, 226, 232-243.

469

470 Laroche, C., Hétu, R., Poirier, S. 1989. The growth of and recovery from TTS in human 471 subjects exposed to impact noise. J Acoust Soc Am, 85, 1681-90.

472

473 Lonsbury-Martin, B., Cutler, W., Martin, G. 1991. Evidence for influence of aging on 474 distortion-product otoacoustic emissions in humans J Acoust Soc Am, 89,1749-1759.

475

476 Lonsbury-Martin, B., Whitehead, M., Martin, G. 1993. Distortion-product otoacoustic 477 emissions in normal and impaired ears: insight into generation processes. Prog Brain Res, 97, $478 \quad 77-90$.

479

480 Lopez-Poveda, E., Johannesen, P. 2009. Otoacoustic emission theories and behavioral 481 estimates of human basilar membrane motion are mutually consistent. J Assoc Res 482 Otolaryngol, 10, 511-523.

483

484 Marshall, L., Lapsley Miller, J., Heller, M. 2001. Distortion-product otoacoustic emissions as 485 a screening tool or noise-induced hearing loss. Noise Health, 3, 12, 43-60. 
487 McBride, D., Williams, S. 2013. Audiometric notch as a sign of noise-induced hearing loss.

488 Occup Environ Med, 2001, 58, 46-51.

489

490 Mulders, W., Robertson, D. 2013. Development of hyperactivity after acoustic trauma in the 491 guinea pig inferior colliculus. Hear Res 298, 104-108.

492

493 Müller, J., Janssen, T. 2008. Impact of occupational noise on pure-tone threshold and 494 distortion product otoacoustic emissions after one workday. Hear Res 246, 9 -22.

495

496 Neely, S, Johnson, T, Kopun, J. 2009. Distortion-product otoacoustic emission I/O 497 characteristics in normal-hearing and hearing-impaired human ears. J Acoust Soc Am, 126, $498 \quad 728-738$.

499 Nelson, D., Nelson, R., Concha-Barrientos, M., Fingerhut, M. 2005.The global burden of 500 occupational noise-induced hearing loss. Am J Ind Med, 48(6), 446-58.

501 NORA National Occupational Research Agenda. 1996. National Institute for Occupational 502 safety and Health. pp 1-73

503

504 Oeken, J., Lenk, A., Bootz, F. Influence of age and presbycusis on DPOAE. Acta 505 Otolaryngol, 2000, 120, 396-403.

506

507 OSHA 1910.95 Occupational Safety and Health Standards available on line at 508 https://www.osha.gov/pls/oshaweb/owadisp.show_document?p_table=STANDARDS\&p_id= 5099735. 
511 Ruggero, M., Rich, N., Recio, A., Narayan, S. 1997. Basilar- membrane responses to tones at

512 the base of the chinchilla cochlea. J Acoust Soc Am, 101, 2151-2163.

513

514 Seixas, N., Kujawa, S., Norton, S., Sheppard, L., Neitzel, R., et al., 2013. Predictors of

515 hearing threshold levels and distortion product otoacoustic emissions among noise exposed

516 young adults. Occup Environ Med, 61, 899-907.

517

518 Syka, J. 2002. Plastic changes in the central auditory system after hearing loss, restoration of 519 function, and during learning. Physiol Rev, 82, 601-628.

520

521 Venet, T., Rumeau, C., Campo, P., Rieger, B., Thomas,A. et al. 2011. Neuronal circuits 522 involved in the middle-ear acoustic ref. Tox Sci,119, 146-155.

Venet, T., Campo, P., Rumeau, C., Eluecque, H., Parietti- Winkler, C. 2012. EchoScan: A new system to objectively assess peripheral hearing disorders. Noise Health, 14, 60, 253-9.

Wagner, W., Heppelmann, G., Müller, J., Janssen, T., Zenner, H.P. 2007. Olivocochlear reflex effect on human distortion product otoacoustic emissions is largest at frequencies with distinct fine structure dips. Hear Res, 223, 83-92.

531 Whitehead, W., McCoy, M., Lonsbury-Martin, B., Martin, G. 1995. Dependence of 532 distortion-product otoacoustic emissions on primary levels in normal and impaired ears. Effects of decreasing L2 below L1. J Acoust Soc Am, 97, 2346-2358. 
Figure legends

537 Figure 1: Pure-tone air-conduction hearing thresholds. Measurements were performed for 538 both groups (noise-exposed vs. control) prior to exposure, i.e., prior to work. Error bars 539 represent the $95 \%$ confidence intervals (Bonferroni).

541 Figure 2: Distortion product otoacoustic emissions obtained at L1=61 dB HL. Measurements were performed for both groups (noise-exposed vs. control) prior to exposure, i.e., before starting work. DP-gram by group. Error bars represent 95\% confidence intervals (Bonferroni).

Figure 3: DPOAE amplitude vs. L1 intensity. (a) DPOAEinput/output curve obtained for primaries with $\mathrm{f} 2=3600 \mathrm{~Hz}$; (b) DPOAEinput/output curve obtained for primaries with $\mathrm{f} 2=$ $4800 \mathrm{~Hz}$; (c) DPOAEinput/output curve obtained for primaries with f2 $=6528 \mathrm{~Hz}$. Measurements for both groups (noise-exposed vs. control) were gathered prior to exposure, i.e., before starting work. Error bars represent 95\% confidence intervals (Bonferroni).

Figure 4: Efferent reflex trigger thresholds at 1000, 2000 and $4000 \mathrm{~Hz}$. DPOAEs were measured in the ipsilateral ear, whereas the suppression noise was delivered through the contralateral ear. Measurements for both groups (noise-exposed vs. control) were performed prior to exposure, i.e., before starting work. Error bars represent $95 \%$ confidence intervals (Bonferroni).

Figure 5: Variations in pure-tone air-conduction hearing thresholds. For noise-exposed and control subjects, the variation was the difference between thresholds measured before and at

559 the end of a workday with a $\mathrm{L}_{\mathrm{EX}, 8 \mathrm{~h}}=85 \mathrm{~dB}(\mathrm{~A})$. Error bars represent $95 \%$ confidence intervals (Bonferroni). * $\mathrm{p} \leq 0.05, * * \mathrm{p} \leq 0.01, * * * \mathrm{p} \leq 0.001$. 
563 Figure 6: Variations in DP-grams. For noise-exposed and control subjects, the difference was

564 calculated between the thresholds measured before and at the end of a workday with a

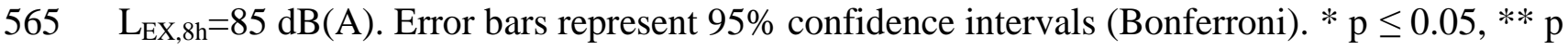

$566 \leq 0.01, * * * \mathrm{p} \leq 0.001$.

567

568 Figure 7: Variations in DPOAE amplitudes. Differences were calculated for measurements

569 performed before and after a working day with a $\mathrm{L}_{\mathrm{EX}, 8 \mathrm{~h}}=85 \mathrm{~dB}(\mathrm{~A})$. (a) DPOAEinput/output

570 variations calculated at $\mathrm{f} 2=3600 \mathrm{~Hz}$; (b) DPOAEinput/output variations calculated at

$571 \mathrm{f} 2=4800 \mathrm{~Hz}$; (c) DPOAEinput/output variations calculated at f2 $=6528 \mathrm{~Hz}$. Error bars represent

$57295 \%$ confidence intervals (Bonferroni). $* \mathrm{p} \leq 0.05, * * \mathrm{p} \leq 0.01, * * * \mathrm{p} \leq 0.001$

573

574 Figure 8: Variation in efferent reflex trigger thresholds at 1000, 2000 and $4000 \mathrm{~Hz}$. DPOAEs

575 were measured in the ipsilateral ear, the suppression noise was delivered through the

576 contralateral ear. The differences were calculated for measurements performed before and

577 after a working day with a $\mathrm{L}_{\mathrm{EX}, 8 \mathrm{~h}}=85 \mathrm{~dB}(\mathrm{~A})$. Error bars represent $95 \%$ confidence intervals

$578 \quad$ (Bonferroni). $* \mathrm{p} \leq 0.05, * * \mathrm{p} \leq 0.01, * * * \mathrm{p} \leq 0.001$ 
580

Table Legends

$581 \quad$ Table 1

582 Cohort of noise-exposed workers. Leq $\mathrm{dB}(\mathrm{A})$ : equivalent continuous noise level measured 583 using the A weighting; $\mathrm{L}_{\mathrm{EX}, 8 \mathrm{~h}}, \mathrm{~dB}(\mathrm{~A})$ : Leq calculated over 8 hours.

584

585

586

587 